\title{
Article \\ Security and Privacy Analysis of Vinoth et al.'s Authenticated Key Agreement Scheme for Industrial IoT
}

\author{
Da-Zhi Sun
}

check for updates

Citation: Sun, D.-Z. Security and Privacy Analysis of Vinoth et al.'s Authenticated Key Agreement Scheme for Industrial IoT. Symmetry 2021, 13, 1952. https://doi.org/ 10.3390/sym13101952

Academic Editors: Weizhi Meng, Georgios Kambourakis and Jun Shao

Received: 15 September 2021

Accepted: 12 October 2021

Published: 16 October 2021

Publisher's Note: MDPI stays neutral with regard to jurisdictional claims in published maps and institutional affiliations.

Copyright: (C) 2021 by the author. Licensee MDPI, Basel, Switzerland. This article is an open access article distributed under the terms and conditions of the Creative Commons Attribution (CC BY) license (https:// creativecommons.org/licenses/by/ $4.0 /)$.
Tianjin Key Laboratory of Advanced Networking (TANK), College of Intelligence and Computing, Tianjin University, Tianjin 300350, China; sundazhi@tju.edu.cn; Tel.: +86-22-2740-1091

\begin{abstract}
Vinoth et al. proposed an authenticated key agreement scheme for industrial IoT (Internet of Things) applications. Vinoth et al.'s scheme aimed to protect the remote sensing data of industrial IoT devices under hostile environments. The scheme is interesting because the authorized user is allowed simultaneously to access the multiple IoT sensing devices. Therefore, we carefully analyzed the security and privacy implications of Vinoth et al.'s scheme. Our findings are summarized as follows. One, Vinoth et al.'s scheme failed to defeat user impersonation attacks. Second, Vinoth et al.'s scheme did not prevent IoT sensing device impersonation attacks. Third, Vinoth et al.'s scheme suffered from replay attacks. Fourth, Vinoth et al.'s scheme was vulnerable to desynchronization attacks. Fifth, Vinoth et al.'s scheme could not maintain user privacy. As a case study, our analysis results enlighten researchers and engineers on the design of robust and efficient authenticated key agreement schemes for IoT applications.
\end{abstract}

Keywords: IoT; sensing networks; authentication; key agreement; impersonation attack; replay attack; desynchronization attack; user privacy

\section{Introduction}

The Internet of Things (IoT) is a fast development in the long and continuing revolution of communications and computing. The IoT has expanded the interconnection of billions of industrial and personal objects through IoT sensing devices, which are typically composed of sensors, actuators, microcontrollers, transceivers, and batteries. IoT sensing devices bound to objects deliver sensor information, act on their environments, and in some cases adapt for the overall management of a larger system, such as a factory [1] or a city [2]. Moreover, these devices always communicate each other and form a remote sensing network. As a typical scenario, Industrial IoT is deployed for achieving intelligent manufacturing because of its advantages in automatic monitoring and efficient control. Under the industrial IoT environment, sensing devices can be remotely accessed and controlled by authorized users. During the process of industrial production, sensing devices collect real-time data. Users obtain this real-time data and then send control commands according to said data.

IoT sensing security [3] is perhaps the most complex and immature area of cybersecurity. The following characteristics hinder secure IoT sensing:

(1) Very large attack surfaces: There is a wide variety of points of vulnerability in IoT sensing systems and a large amount of data that may be compromised.

(2) Widespread deployment: There is ongoing, rapid deployment of IoT arrangements in commercial and industrial environments and, more importantly, in critical infrastructure environments. Most IoT sensing devices are remote and out of control. These deployments are attractive targets for security attacks.

(3) Constrained device resources: IoT sensing devices are typically constrained, with limited memory, processing power, and power supply. 
(4) Low cost: IoT sensing devices are always manufactured, purchased, and deployed in the millions. This fact provides great incentive for manufacturers and customers to minimize the cost of these devices.

Motivation of This Paper. In the normal course of things, the user requires simultaneously access to multiple IoT sensing devices for a complex industrial task. Because of serious security and privacy threats, IoT sensing devices, especially remote devices, are required to support mutual authentication and secret key establishment with their users. The authenticated key agreement scheme provides authentication and key establishment services among users and multiple IoT sensing devices. We therefore analyzed the security and privacy of the authenticated key agreement scheme. Our research focused on not only outside but inside attackers, i.e., malicious users and corrupt IoT sensing devices.

\subsection{Industrial IoT Sensing Model and Its Authenticated Key Agreement Scheme}

In this section, we describe the sensing model and authenticated key agreement scheme studied in this paper.

\subsubsection{Industrial IoT Sensing Model}

The sensing model is depicted in Figure 1. There are three categories of entities, i.e., gateway nodes (GWNs), users, and industrial IoT sensing devices.

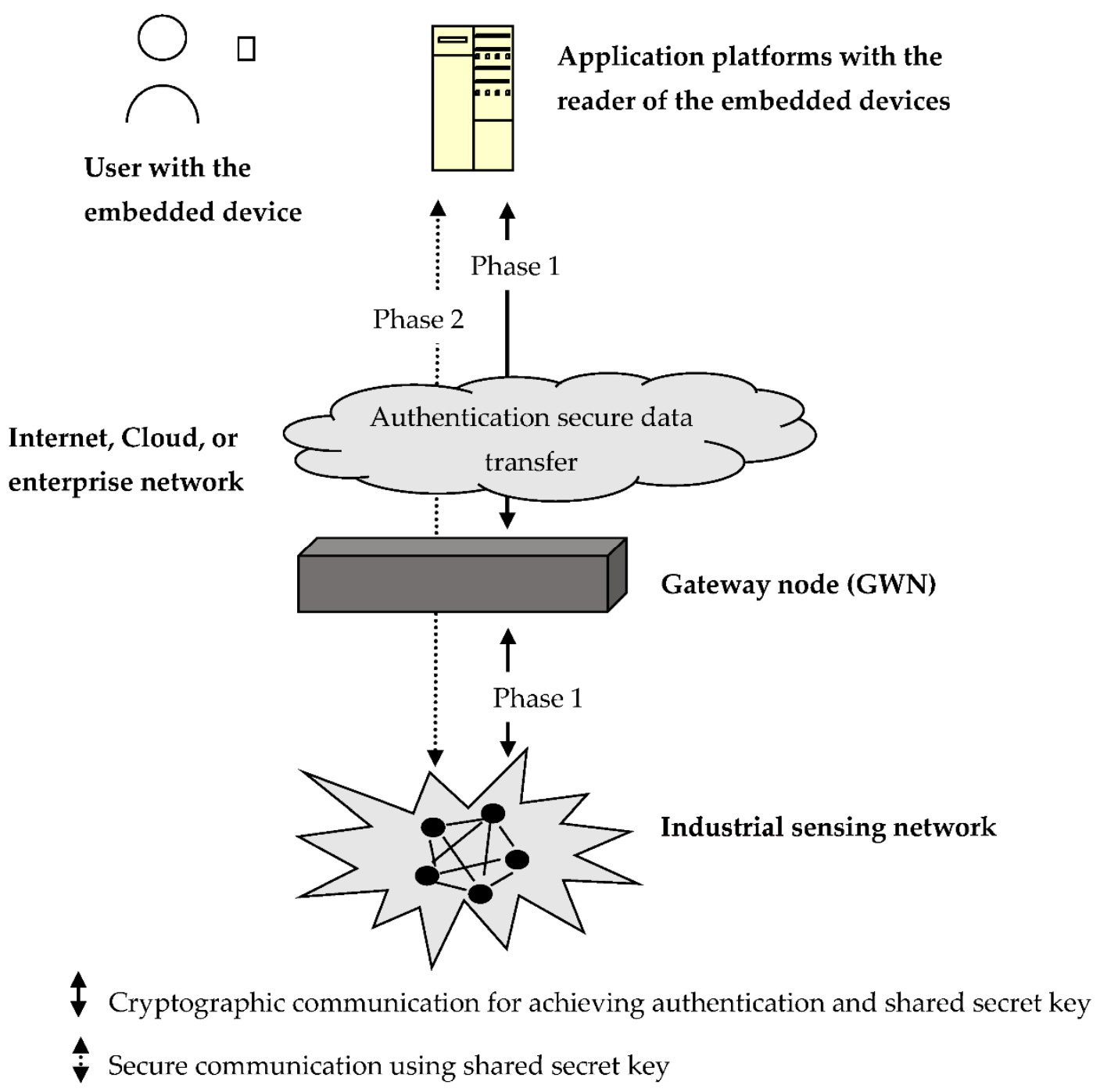

Figure 1. Industrial IoT sensing model. 
(1) GWN: GWNs interconnect IoT sensing devices with high-level communication networks and perform the necessary translation between the protocols used in communication networks and those used in IoT sensing devices.

(2) Users: The users are allowed to access IoT sensing devices through GWNs. They gain security and privacy services with the help of embedded devices such as smart cards.

(3) IoT sensing devices: IoT sensing devices are utilized to monitor the status of objects and collect the information stored therein. Users can obtain the information collected by these devices in real time.

In our industrial IoT sensing model, we assumed that the users and the IoT sensing devices were untrusted entities. GWNs [4], meanwhile, cannot be compromised and were therefore considered to be fully trusted by the users and the IoT sensing devices. This assumption is reasonable because GWNs are usually placed in secure environments and equipped with tamper-resistant devices.

\subsubsection{Authenticated Key Agreement Scheme}

To set up a secure sensing network, the GWN initially writes some authentication credentials into IoT sensing devices. The user first registers to the GWN, and both the user and GWN write the authentication credentials into the user's embedded device. When the registered users want to access the deployed IoT sensing devices, they run an authentication session using their embedded device. During the authentication session, GWN helps the user and the IoT sensing devices authenticate each other and establish a shared secret key for subsequent secure communication. In addition, the users can change their authentication credentials, and the GWN can allow new IoT sensing devices to join the deployed sensing network and revoke existing devices from said network.

However, an attacker may exploit the vulnerabilities in the authenticated key agreement scheme to perform attacks, because the messages of the authentication session are often transmitted through a public channel, and this brings security problems in the industrial IoT environment. It is possible for an inside or outside attacker to impersonate an authorized user to obtain data by accessing sensing devices or to impersonate a legal IoT sensing device to provide fake data. These unsatisfactory security risks could lead to the destruction of industrial activity.

\subsection{Related Work}

In recent years, many authenticated key agreement schemes [5,6] have been proposed for IoT remote sensing environments, such as industrial IoT, telemedicine, and smart home. We review previous work on four dimensions.

From the user credentials perspective, authenticated key agreement schemes are classified into two categories, i.e., two- and three-factor (multifactor) schemes. In twofactor schemes [7-15], the security of the user is protected by both the secret key stored in the smart card and the human-memorizable password, and the user applies the password and the smart card to complete the authentication session. Compared to two-factor schemes, three-factor schemes [4,16-22] add biometrics to the user credentials; that is, the user must provide the smart card, the password, and biometrics at the same time.

In many privacy applications, the users does not want authentication sessions to be associated with their identity. This means that the user's identity is disclosed only to an authorized set of GWN and IoT sensing devices during the authentication sessions. Therefore, to preserve user privacy, authenticated key agreement schemes [23-27] thwart attempts to disclose or link users' identities by exploiting their authentication sessions.

Many researchers have extended authenticated key agreement schemes [28-34] to multi-gateway IoT environments. These revised schemes provide the user with a single sign under a set of GWNs. That is, when the user is authenticated by a GWN in the set of GWNs, he/she can access all IoT sensing devices governed by the set of GWNs even if the devices in question are not directly managed by the specific GWN that authenticated the 
user. In addition, the multi-gateway schemes can solve the packet-collision problem due to single GWN mode.

Users often access multiple IoT sensing devices to complete complex tasks. It is inefficient for the user to run a separate authentication session with each IoT sensing device. Moreover, the logical relevance of the authentications and the shared secret keys cannot be guaranteed if the user independently runs several authentication sessions for a task. Hence, some authenticated key agreement schemes $[4,35,36]$ have recently begun to provide authentication and group secret-key establishment between the user and multiple IoT sensing devices in an authentication session.

\subsection{Our Contributions}

In the IEEE Internet of Things Journal, Vinoth et al. [4] proposed an authenticated key agreement scheme that aimed to protect the remote sensing data of industrial IoT under the hostile environments. We carefully analyzed security and privacy under Vinoth et al.'s scheme. Our results are as follows.

(1) Vinoth et al.'s scheme failed to defeat a user impersonation attack. A legal but malicious user could impersonate IoT sensing devices, other users, and the GWN.

(2) Vinoth et al.'s scheme did not prevent IoT sensing device impersonation attacks. A legal but corrupt IoT sensing device can impersonate users, the GWN, and other IoT sensing devices.

(3) Vinoth et al.'s scheme suffered from replay attacks. Attackers can reuse the previous message in the authentication session to cheat the user and the GWN.

(4) Vinoth et al.'s scheme was vulnerable to desynchronization attacks. In these attacks, an attacker induces an inconsistent internal status between the user and the GWN. This security flaw causes the GWN to deny the service for the user.

(5) Vinoth et al.'s scheme cannot maintain user privacy. User identity is compromised during the run of the authentication session.

As a matter of convenience, in Table 1, we list some notation used throughout our paper.

Table 1. Description of notations.

\begin{tabular}{|c|c|}
\hline Term & Definition \\
\hline GWN, U & Gateway node and user \\
\hline $\mathrm{S}_{j}$ & jth IoT sensing device \\
\hline$I D_{G W N}, I D_{U}, I D_{S_{j}}$ & GWN's, U's, and $S_{j}$ 's identities \\
\hline$T I D_{U}$ & U's temporary identity for user anonymity \\
\hline$\gamma, K_{G W N}$ & GWN's long-term secret keys \\
\hline $\begin{array}{l}K_{G W N-U} \\
P W\end{array}$ & $\begin{array}{l}\text { Long-term secret key shared by GWN and U } \\
\text { U's password }\end{array}$ \\
\hline$B, B K, \tau$ & $\begin{array}{l}\text { U's biometrics, biometrics key, and public } \\
\text { reproduction parameter }\end{array}$ \\
\hline$s_{j}, f_{j}, k_{j}$ & $\mathrm{~S}_{j}{ }^{\prime}$ s secret parameters \\
\hline$K_{G W N-S_{j}}$ & Secret key shared by GWN and $S_{j}$ \\
\hline$K_{U-S j}$ & Secret session key shared by $U$ and $S_{j}$ \\
\hline$r_{G W N}, r_{U}, R N$ & Random numbers \\
\hline$T S_{1}, T S_{2}, T S_{3}, T S_{4}, T S_{1}{ }^{\prime}, T S_{2}{ }^{\prime}, T S_{3}{ }^{\prime}, T S_{4}{ }^{\prime}$ & Timestamps \\
\hline$\Delta T S$ & Maximum transmission delay \\
\hline$\varphi()$ & Vinoth et al.'s access structure function [4] \\
\hline $\operatorname{Gen}() / \operatorname{Rep}()$ & $\begin{array}{c}\text { Generation algorithm/reproduction algorithm } \\
\text { using biometrics fuzzy extractor }\end{array}$ \\
\hline$h()$ & Cryptographic hash function \\
\hline$E_{K}() / D_{K}()$ & $\begin{array}{c}\text { Encryption algorithm/decryption algorithm } \\
\text { using secret key } K\end{array}$ \\
\hline $\bmod$ & Congruent \\
\hline$\oplus, \|$ & Bitwise exclusive-or and concatenation \\
\hline
\end{tabular}




\section{Review of Vinoth et al.'s Authenticated Key Agreement Scheme}

\subsection{Scheme Description}

Vinoth et al.'s scheme is composed of seven phases: the offline sensing device registration phase, the user registration phase, the login phase, the authenticated key agreement phase, the biometrics and password update phase, the dynamically sensing device joining phase, and the sensing device revocation phase. For a self-contained discussion, we review the first four phases, which are related to our discussion. The full technical details of Vinoth et al.'s scheme can be found in [4].

\subsubsection{Offline Sensing Device Registration Phase}

GWN picks a unique $I D_{S_{j}}$ for $S_{j}$, where $j=1,2, \ldots, n$. GWN then chooses a $K_{G W N-S_{j}}$ and two $n$-dimensional vectors Vector ${ }_{1}$ and Vector $_{2}$ such that $K_{G W N-S_{j}}=$ Vector $_{1} \cdot x_{0}$ and $K_{G W N-s_{j}}{ }^{2}=$ Vector $_{2} \cdot x_{0}$, where $x_{0}=\varphi(\mathrm{GWN})$. GWN calculates $s_{j}=$ Vector $_{1} \cdot x_{j}$ and $f_{j}=$ Vector $_{2} \cdot x_{j}$, where $x_{j}=\varphi\left(S_{j}\right)(1 \leq j \leq n)$. GWN computes and stores $\lambda_{t}$, where $K_{G W N-S_{j}=\sum_{t=1}^{n} \lambda_{t} s_{t}}$

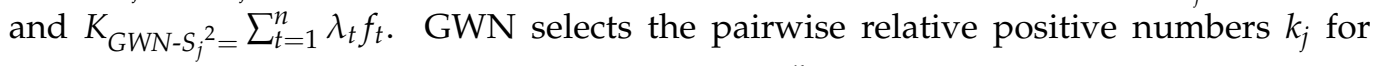
each $S_{j}(1 \leq j \leq n)$. GWN computes $M u l=\prod_{j=1}^{n} k_{j}$ and $M u l_{j}=M u l / k_{j}$ and generates a random number Nonce $_{j}$ such that $\mathrm{Mul}_{j} \times$ Nonce $_{j} \equiv 1 \bmod k_{j}$. GWN computes $\gamma=\sum_{j=1}^{n} \operatorname{Var}_{j}=\sum_{j=1}^{n} M u l_{j} \times$ Nonce $_{j}$ and stores $\gamma$. GWN securely sends $I D_{S_{j}}, s_{j}, f_{j}$, and $k_{j}$ to each $\mathrm{S}_{j}(1 \leq j \leq n)$, and then $\mathrm{S}_{j}$ stores them. In the end, GWN deletes other messages.

\subsubsection{User Registration Phase}

Step 1: $U$ chooses a unique $I D_{U}$ and a $P W$, imprints the $B$, and computes $(B K, \tau)=\operatorname{Gen}(B)$. $\mathrm{U}$ generates a random 128-bit number $a$, calculates $T P W=h\left(I D_{U}\|P W\| B K\right) \oplus a$, and securely sends the message $<I D_{U}, T P W>$ to GWN.

Step 2: When receiving $\left\langle I D_{U}, T P W>\right.$, GWN randomly generates a 1024-bit $K_{G W N}$ and a 128-bit $T I D_{U}$, and then computes $K_{G W N-U}=h\left(I D_{U} \| K_{G W N}\right), A=K_{G W N-U} \oplus T P W$, and $C=I D_{G W N} \oplus T P W$. GWN stores $T I D_{U}, I D_{U}$, and $K_{G W N-U}$ in its database. Finally, GWN writes $\left\{T I D_{U}, A, C, h()\right\}$ into a smart card and securely sends the card to $\mathrm{U}$.

Step 3: When receiving the card, $\mathrm{U}$ computes $R P W=h\left(I D_{U}\|P W\| B K\right), A^{\prime}=A \oplus T P W \oplus R P W$, $D=a \oplus h\left(I D_{U} \| B K\right), C^{\prime}=C \oplus T P W \oplus h\left(I D_{U} \| B K\right)$, and $V \equiv h\left(R P W\|A\| a \| h\left(I D_{U} \| B K\right)\right) \bmod \omega$, where $\omega$ is the medium integer [37-39]. Finally, $\mathrm{U}$ rewrites $\left\{T I D_{U}, A^{\prime}, C^{\prime}, D, V, \operatorname{Gen}(), \operatorname{Rep}()\right.$, $h(), \tau, \omega\}$ into the card.

\subsubsection{Login Phase}

Step 1: $U$ inserts the smart card into the card reader, and then further inputs the $I D_{U}$ and $P W$ and imprints the $B$. The smart card reconstructs $B K=\operatorname{Rep}(B, \tau)$ and computes $R P W=h\left(I D_{U}\|P W\| B K\right), a=D \oplus h\left(I D_{U} \| B K\right)$, and $A=A^{\prime} \oplus a$. The smart card further checks whether $V \equiv h\left(R P W\|A\| a \| h\left(I D_{U} \| B K\right)\right) \bmod \omega$. If not, the smart card terminates the login request.

Step 2: The smart card generates $r_{U}$ and obtains current $T S_{1}$. The smart card computes $I D_{G W N}=C^{\prime} \oplus h\left(I D_{U} \| B K\right), M_{1}=A^{\prime} \oplus R P W \oplus r_{U}$, and $M_{2}=h\left(T I D_{U}\left\|M_{1}\right\| I D_{G W N}\left\|r_{U}\right\| T S_{1}\right)$. In the end, the smart card sends the message $<T I D_{U}, M_{1}, M_{2}, T S_{1}>$ to GWN.

\subsubsection{Authenticated Key Agreement Phase}

Step 1: After receiving $\left\langle T I D_{U}, M_{1}, M_{2}, T S_{1}>\right.$, GWN obtains the current $T S_{1}$ 'and checks the freshness of the login request by verifying whether $\left|T S_{1}-T S_{1}{ }^{\prime}\right| \leq \Delta T S$. If not, GWN terminates this session. GWN searches its database by using the keyword $T I D_{U}$ and retrieves $I D_{U}$ and $K_{G W N-U}$. GWN calculates $r_{U}=M_{1} \oplus K_{G W N-U}$ and checks whether $M_{2}=h\left(T I D_{U}\left\|M_{1}\right\| I D_{G W N}\left\|r_{U}\right\| T S_{1}\right)$. If not, GWN terminates this session. GWN generates $r_{G W N}\left(r_{G W N} \leq \min \left\{k_{j}\right\}, j=1,2, \ldots, n\right)$ and obtains the current $T S_{2}$. GWN computes $M_{3}=r_{G W N} \times \gamma, M_{4}=E_{r G W N}\left(I D_{U}, I D_{G W N}, r_{U}, r_{G W N} \oplus K_{G W N-U}\right)$, and $M_{5}=h\left(I D_{U}\left\|I D_{G W N}\right\| r_{U}\left\|M_{3}\right\| K_{G W N-U} \| T S_{2}\right)$. Finally, GWN broadcasts the message $<M_{3}, M_{4}$, $M_{5}, T S_{2}>$ to all $S_{j}$ s. 
Step 2: When receiving $<M_{3}, M_{4}, M_{5}, T S_{2}>$, each $S_{j}$ obtains current $T S_{2}{ }^{\prime}$ and checks the freshness of the message by verifying whether $\left|T S_{2}-T S_{2}{ }^{\prime}\right| \leq \Delta T S$. If not, $S_{j}$ terminates this session. $S_{j}$ computes $r_{G W N} \equiv M_{3} \bmod k_{j}$ and $\left(I D_{U}, I D_{G W N}, r_{U}, r_{G W N} \oplus K_{G W N-U}\right)=D_{r G W N}\left(M_{4}\right)$, and further checks whether $M_{5}=h\left(I D_{U}\left\|I D_{G W N}\right\| r_{U}\left\|M_{3}\right\| r_{G W N} \oplus K_{G W N-U} \oplus r_{G W N} \| T S_{2}\right)$. If not, $S_{j}$ terminates this session. $S_{j}$ computes $M_{6}=E_{r G W N}\left(I D_{S_{j}}, s_{j}, f_{j}\right)$ and obtains current $T S_{3}$. $S_{j}$ returns the message $<M_{6}, T S_{3}>$ to $G W N$.

Step 3: When receiving $<M_{6}, T S_{3}>$, GWN obtains current $T S_{3}{ }^{\prime}$ and checks the freshness of the message by verifying whether $\left|T S_{3}-T S_{3}{ }^{\prime}\right| \leq \Delta T S$. If not, GWN terminates the session. GWN computes $\left(I D_{S_{j}}, s_{j}, f_{j}\right)=D_{r G W N}\left(M_{6}\right)$ from each $S_{j}$. GWN calculates $\theta_{1}=\sum_{t=1}^{n} \lambda_{t} s_{t}$ and $\theta_{2}=\sum_{t=1}^{n} \lambda_{t} f_{t}$ and checks whether $\theta_{1}^{2}=\theta_{2}$. If not, GWN terminates this session. GWN views $\theta_{1}$ as $K_{G W N-S_{j}}$ and computes $M_{7}=h\left(K_{G W N-S_{j}} \| r_{G W N}\right), M_{8}=M_{7} \times \gamma$, and $M_{9}=h\left(M_{7} \| M_{8}\right)$. Moreover, GWN generates a new temporary identity $\operatorname{TID}_{U}{ }^{\text {new }}$, obtains the current $T S_{4}$, and computes $M_{10}=E_{K G W N-U}\left(r_{G W N}, r_{U}, M_{7}\right), M_{11}=h\left(I D_{U}\left\|K_{G W N-U}\right\| T S_{4}\right) \oplus T I D_{U}{ }^{\text {new }}$, and $M_{12}=h\left(M_{10}\left\|M_{7}\right\| r_{U}\right)$. In the end, GWN broadcasts the message $\left\langle M_{8}, M_{9}>\right.$ to all $S_{j} \mathrm{~s}$ and sends the message $<M_{10}, M_{11}, M_{12}, T S_{4}>$ to $U$.

Step 4: When receiving $\left\langle M_{8}, M_{9}>\right.$, each $S_{j}$ calculates $M_{7} \equiv M_{8} \bmod k_{j}$ and checks whether $M_{9}=h\left(M_{7} \| M_{8}\right)$. If not, $S_{j}$ terminates this session. $S_{j}$ computes $K_{U-S j}=h\left(I D_{U} \|\right.$ $\left.I D_{G W N}\left\|r_{G W N}\right\| r_{U}\left\|M_{7}\right\| K_{G W N-U}\right)$ and $M_{13}=h\left(K_{U-S j}\left\|I D_{G W N}\right\| I D_{U}\right)$, and then sends the message $<M_{13}>$ to $\mathrm{U}$.

Step 5: When receiving $<M_{10}, M_{11}, M_{12}, T S_{4}>, \mathrm{U}$ obtains the current $T S_{4}{ }^{\prime}$ and checks the freshness of the message by verifying whether $\left|T S_{4}-T S_{4}{ }^{\prime}\right| \leq \Delta T S$. If not, $U$ terminates this session. U computes $\left(r_{G W N}, r_{U}, M_{7}\right)=D_{K G W N-U}\left(M_{10}\right)$. Then, $U$ checks whether the decrypted $r_{U}$ is equal to the local $r_{U}$ and $M_{12}=h\left(M_{10}\left\|M_{7}\right\| r_{U}\right)$. If any one of them is unequal, $\mathrm{U}$ terminates this session. U calculates $K_{U-S j}=h\left(I D_{U}\left\|I D_{G W N}\right\| r_{G W N}\left\|r_{U}\right\| M_{7} \| K_{G W N-U}\right)$. Furthermore, when receiving $<M_{13}>$ from each $S_{j}$, $U$ checks whether $M_{13}=h\left(K_{U-S j}\left\|I D_{G W N}\right\| I D_{U}\right)$. If not, $\mathrm{U}$ terminates this session. $\mathrm{U}$ computes $T I D_{U}{ }^{\text {new }}=h\left(I D_{U}\left\|K_{G W N-U}\right\| T S_{4}\right) \oplus M_{11}$ and replaces $T I D_{U}$ with $T I D_{U}{ }^{\text {new }}$.

Figure 2 shows the process of Vinoth et al.'s login phase and authenticated key agreement phase.

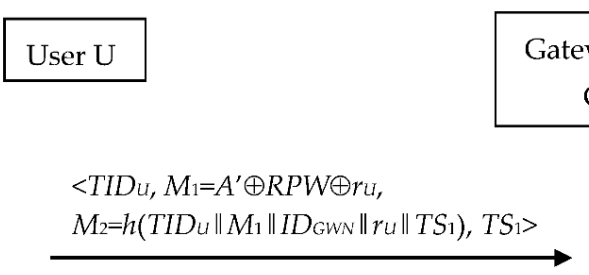

Sensing Device $S_{\text {f }}$

Sensing Device

\section{GWN}

$<T I D_{u}, M_{1}=A^{\prime} \oplus R P W \oplus r u$,
$M_{2}=h\left(T I D u\left\|M_{1}\right\| I D_{G W N}\|r u\| T S_{1}\right), T S_{1}>$

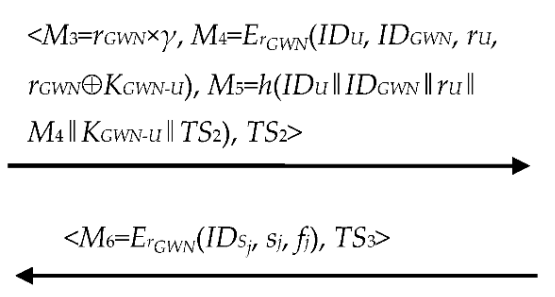

$<M_{10}=E_{K G W N-U}\left(r_{G W N}, r_{U}, M_{7}\right)$,

$M_{11}=h\left(I D u\left\|K_{G W N-u}\right\| T S_{4}\right) \oplus T I D^{\text {nerv }}$, $M_{12}=h\left(M_{10}\left\|M_{7}\right\| r u\right), T S_{4}>$

$<M_{8}=M_{7} \times \gamma, M_{9}=h\left(M_{7} \| M_{8}\right)>$

$<M_{13}=h\left(K_{u-S_{j}}\left\|I D_{\mathrm{GWN}}\right\| I D u\right)>$

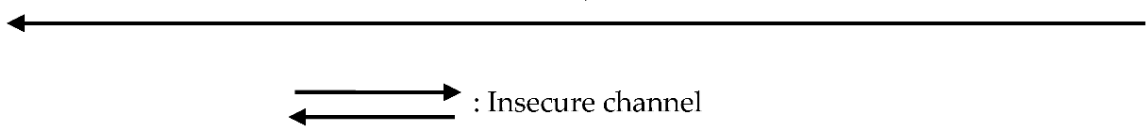

Figure 2. Vinoth et al.'s login phase and authenticated key agreement phase. 


\subsection{Vinoth et al.'s Security Assumption}

Vinoth et al. claimed that their scheme was secure under the Canetti-Krawczyk threat model [40], which assumes that an attacker can eavesdrop on, intercept, modify, forge, and delete messages transmitted between any two entities over the public channel. An attacker can also impersonate users, IoT sensing devices, and the GWN to receive and send the messages. Furthermore, the attacker has the capability to expose some secrets of the users and the IoT sensing devices. More importantly, the attacker can be an insider, i.e., a user or an IoT sensing device, because users and sensing devices are untrusted entities. Under the Canetti-Krawczyk threat model, we discuss five types of attacks on Vinoth et al.'s scheme.

\section{User Impersonation Attack}

We showed that Vinoth et al.'s scheme was vulnerable to user impersonation attacks. That is, a legal but malicious user could impersonate IoT sensing devices, any other user, and the GWN in the deployed network. We assume that $\mathrm{U}_{a}$ is a legal but malicious user in Vinoth et al.'s scheme and maintains the identity $I D_{U a}$, temporary identity $T I D_{U a}$, and long-term secret key $K_{G W N-U a}$ shared with GWN.

\subsection{Impersonation of IoT Sensing Devices}

To impersonate a target $S_{j}, U_{a}$ first initiates his/her authentication session with GWN. In Steps 2 and 3 of the authenticated key agreement phase, $\mathrm{U}_{a}$ eavesdrops on GWN's message $<M_{6}, T S_{3}>$ from $S_{j}$ and $S_{j}{ }^{\prime} s<M_{8}, M_{9}>$ from GWN. When $\mathrm{U}_{a}$ receives the message $<M_{10}, M_{11}, M_{12}, T S_{4}>$ in Step 3 of the authenticated key agreement phase, $\mathrm{U}_{a}$ computes $\left(r_{G W N}, r_{U}, M_{7}\right)=D_{K G W N-U a}\left(M_{10}\right)$. Now, $\mathrm{U}_{a}$ is able to compute $\left(I D_{S_{j}}, s_{j}, f_{j}\right)=D_{r G W N}\left(M_{6}\right)$ and derive $\gamma$ by evaluating $M_{8} / M_{7}$.

Figure 3 illustrates that $\mathrm{U}_{a}$ impersonates $S_{j}$ using $\gamma, I D_{S_{j}}, s_{j}$, and $f_{j}$ and cheats the GWN and any other $U$ during an authentication session. In Step 2 of the authenticated key agreement phase, $\mathrm{U}_{a}$ uses $M_{3} / \gamma$ instead of $M_{3} \bmod k_{j}$ to recover $r_{G W N}$. In Step 4 of the authenticated key agreement phase, $\mathrm{U}_{a}$ uses $M_{8} / \gamma$ instead of $M_{8} \bmod k_{j}$ to recover $M_{7}$ Other operations of $\mathrm{U}_{a}$ and $S_{j}$ are exactly the same. After the authentication session, $\mathrm{U}$ shares $K_{U-S j}=h\left(I D_{U}\left\|I D_{G W N}\right\| r_{G W N}\left\|r_{U}\right\| M_{7} \| K_{G W N-U}\right)$ with $\mathrm{U}_{a}$ instead of $S_{j}$ and updates a new temporary identity $T_{I} D^{\text {new }}$.

\subsection{Impersonation of Other Users}

Assume that any other user $U$ runs the login phase and authenticated key agreement phase. In Step 1 and Step 3 of the authenticated key agreement phase, $\mathrm{U}_{a}$ eavesdrops on $S_{j}$ 's message $<M_{3}, M_{4}, M_{5}, T S_{2}>$ from GWN and U's message $<M_{10}, M_{11}, M_{12}, T S_{4}>$ from GWN. From Section 3.1, we know that $\mathrm{U}_{a}$ obtains GWN's $\gamma$. Hence, $\mathrm{U}_{a}$ computes $r_{G W N}=M_{3} / \gamma$ and $\left(I D_{U}, I D_{G W N}, r_{U}, r_{G W N} \oplus K_{G W N-U}\right)=D_{r G W N}\left(M_{4}\right)$. Since $U_{a}$ has U's $I D_{U}$ and $K_{G W N-U}, U_{a}$ can further compute U's new temporary identity $T I D_{U}{ }^{\text {new }}$ by computing $h\left(I D_{U}\left\|K_{G W N-U}\right\| T S_{4}\right) \oplus M_{11}$. Now, $U_{a}$ can exploit U's $I D_{U}, K_{G W N-U}$, and $T I D_{U}{ }^{\text {new }}$ to impersonate $U$ in a new authentication session.

\subsection{Impersonation of $G W N$}

$\mathrm{U}_{a}$ can impersonate $\mathrm{GWN}$ to cheat $\mathrm{U}$ and $S_{j}$. First, $\mathrm{U}_{a}$ obtains $I D_{U}, K_{G W N-U}, I D_{G W N}$, and $\gamma$ as described in Section 3.2. Figure 4 shows how $U_{a}$ impersonates GWN. In Step 3 of the authenticated key agreement phase, $\mathrm{U}_{a}$ neither decrypts $M_{6}$, retrieves $K_{G W N-S_{j}}$, nor computes $M_{7}=h\left(K_{G W N-S_{j}} \| r_{G W N}\right)$. Instead, $\mathrm{U}_{a}$ directly replaces $M_{7}$ with his/her random $R N$. Note that both $U$ and $S_{j}$ should authenticate each other and share $K_{U-S j}=h\left(I D_{U}\left\|I D_{G W N}\right\| r_{G W N}\left\|r_{U}\right\| R N \| K_{G W N-U}\right)$, because they do not check the validity of $M_{7}$. 


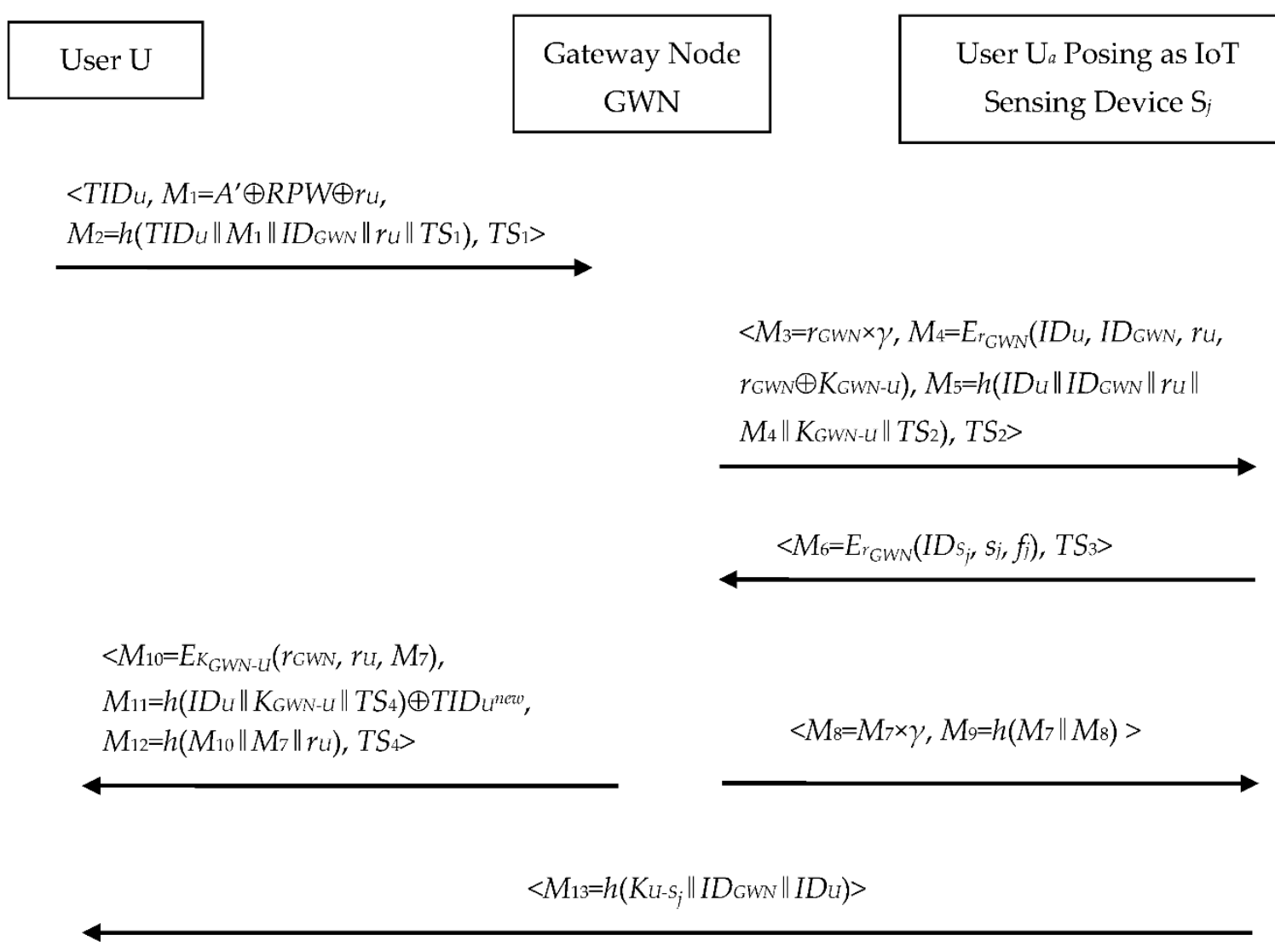

Figure 3. User impersonation attack on IoT sensing devices.

User U

$<T I D u, M_{1}=A^{\prime} \oplus R P W \oplus r u$,

$M_{2}=h\left(T I D u\left\|M_{1}\right\| I D_{G W N}\|r u\| T S_{1}\right), T S_{1}>$
IoT Sensing Device $\mathrm{S}_{j}$
$<M_{10}=E_{K_{G W N}-u}\left(r_{G W N}, r u, R N\right)$,

$M_{11}=h\left(I D u\left\|K_{G W N-u}\right\| T S_{4}\right) \oplus T I D u^{\text {new }}$, $M_{12}=h\left(M_{10}\|R N\| r u\right), T S_{4}>$
User $\mathrm{U}_{a}$ Posing as

Gateway Node GWN
$<M_{3}=r_{G W N} \times \gamma, M_{4}=E_{r_{G W N}}\left(I D u, I D_{G W N}, r u\right.$, $r_{G W N} \oplus K_{G W N-U),} M_{5}=h\left(I D u\left\|I D_{G W N}\right\| r u \|\right.$ $\left.M_{4}\left\|K_{G W N-u}\right\| T S_{2}\right), T S_{2}>$

$<M_{6}=E_{r_{G W N}}\left(I D s_{j}, s_{j}, f_{j}\right), T S_{3}>$

$<M_{8}=R N \times \gamma, M_{9}=h\left(R N \| M_{8}\right)>$

$$
<M_{13}=h\left(K u-S_{j}\left\|I D_{G W N}\right\| I D U\right)>
$$

Figure 4. User impersonation attack on the GWN. 


\subsection{Further Disscussion}

In every authentication session of Vinoth et al.'s scheme, the GWN uses its long-term secret key $\gamma$ to secure its short-term secret key $r_{G W N}$ for each user and each IoT sensing device. However, any user can directly recover $\gamma$ after an authentication session. Hence, the user derives all the secrets of other users, the GWN, and IoT sensing devices and implements the impersonation attacks. To defeat a user's impersonation attack, $\gamma$ cannot be disclosed to users.

User impersonation attacks are a serious threat under industrial IoT environments. Malicious users may impersonate other, honest users to collect sensitive industrial data or set dangerous processing instructions. By impersonating IoT sensing devices, malicious users can provide fake industrial data to other users. If malicious users employ impersonation of the GWN, they can manipulate a secure connection between the target user and IoT sensing devices. That is, malicious users can decide which IoT sensing devices can be connected to the target user.

\section{IoT Sensing Device Impersonation Attacks}

We showed that Vinoth et al.'s scheme was vulnerable to IoT sensing device impersonation attacks. That is, any legal but corrupt sensing device could impersonate users, the GWN, and any other IoT sensing devices in the deployed network. We assumed that $S_{j}$ is a legal but corrupt IoT sensing device.

\subsection{Impersonation of Users}

To obtain $T I D_{U}, S_{j}$ eavesdrops on GWN's message $<T I D_{U}, M_{1}, M_{2}, T S_{1}>$ during Step 2 of the login phase. $S_{j}$ further obtains U's $I D_{U}, I D_{G W N}$, and $K_{G W N-U}$ in Step 2 of the authenticated key agreement phase. However, $S_{j}$ does not return the message $<M_{6}, T S_{3}>$ to GWN. In this situation, both $\mathrm{U}$ and GWN terminate this session and therefore fail to update $T I D_{U}$. Alternatively, $S_{j}$ returns the message $<M_{6}, T S_{3}>$ to GWN in Step 2 of the authenticated key agreement phase and further eavesdrops on U's message $<M_{10}, M_{11}$, $M_{12}, T S_{4}>$ during Step 3 of the authenticated key agreement phase. At this time, $S_{j}$ further obtains TID ${ }^{\text {new }}$ by computing $h\left(I D_{U}\left\|K_{G W N-U}\right\| T S_{4}\right) \oplus M_{13}$. Now, $S_{j}$ knows all of U's secrets. As shown in Figure 5, $S_{j}$ can start a new authentication session and perform the following steps to impersonate $\mathrm{U}$ :

(1) In Step 2 of login phase, $S_{j}$ uses $K_{G W N-U}, T I D_{U}$, and $I D_{G W N}$ to generate $M_{1}$ and $M_{2}$.

(2) In Step 5 of authenticated key agreement phase, $S_{j}$ does exactly the same as $U$.

At the end of the authentication session, $S_{m}(1 \leq m \neq j \leq n)$ authenticates $S_{j}$ as $U$ and shares $K_{U-S j}=h\left(I D_{U}\left\|I D_{G W N}\right\| r_{G W N}\left\|r_{U}\right\| M_{7} \| K_{G W N-U}\right)$ with $S_{j}$.

\subsection{Impersonation of $G W N$}

Moreover, in Step 2 of the authenticated key agreement phase, $S_{j}$ can use $k_{j}$ to compute $r_{G W N} \equiv M_{3}$ mod $k_{j}$. Hence, $S_{j}$ can further derive GWN's $\gamma$ by computing $M_{3} / r_{G W N}$. Now, $S_{j}$ can exploit $T I D_{U}, I D_{U}, K_{G W N-U}, I D_{G W N}$, and $\gamma$ to impersonate GWN. As shown in Figure 6, the fake GWN impersonated by $S_{j}$ omits $M_{6}$, generates its own $R N$, and then replaces $M_{7}$ with $R N$. Both $U$ and $S_{j}$ believe that $R N$ is a legal $M_{7}$ because they do not check the validity of $M_{7}$. Finally, both $U$ and $S_{m}$ authenticate each other and share $K_{U-S j}=h\left(I D_{U}\left\|I D_{G W N}\right\| r_{G W N}\left\|r_{U}\right\| R N \| K_{G W N-U}\right)$. 


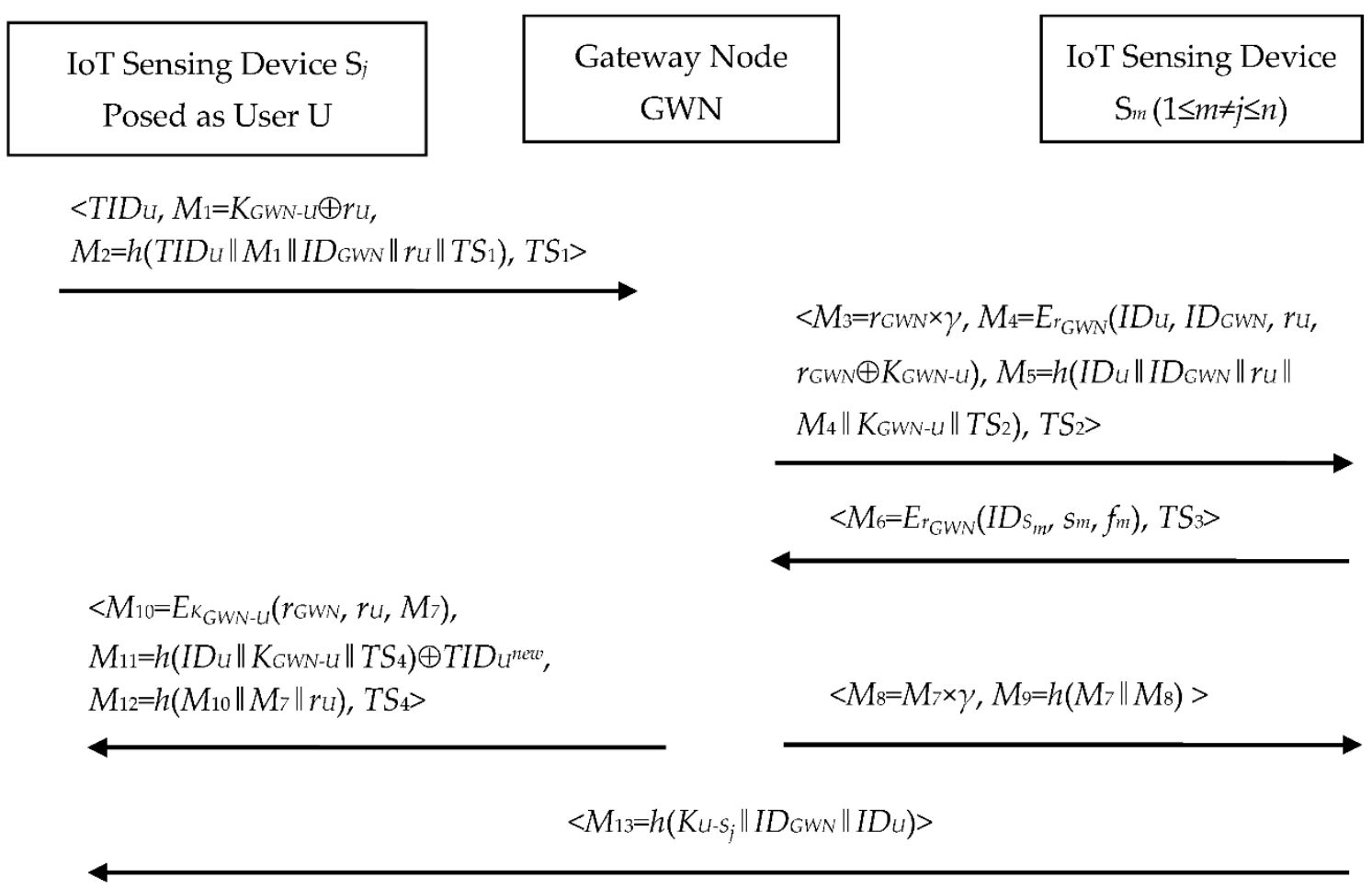

Figure 5. IoT sensing device impersonation attack on a user.

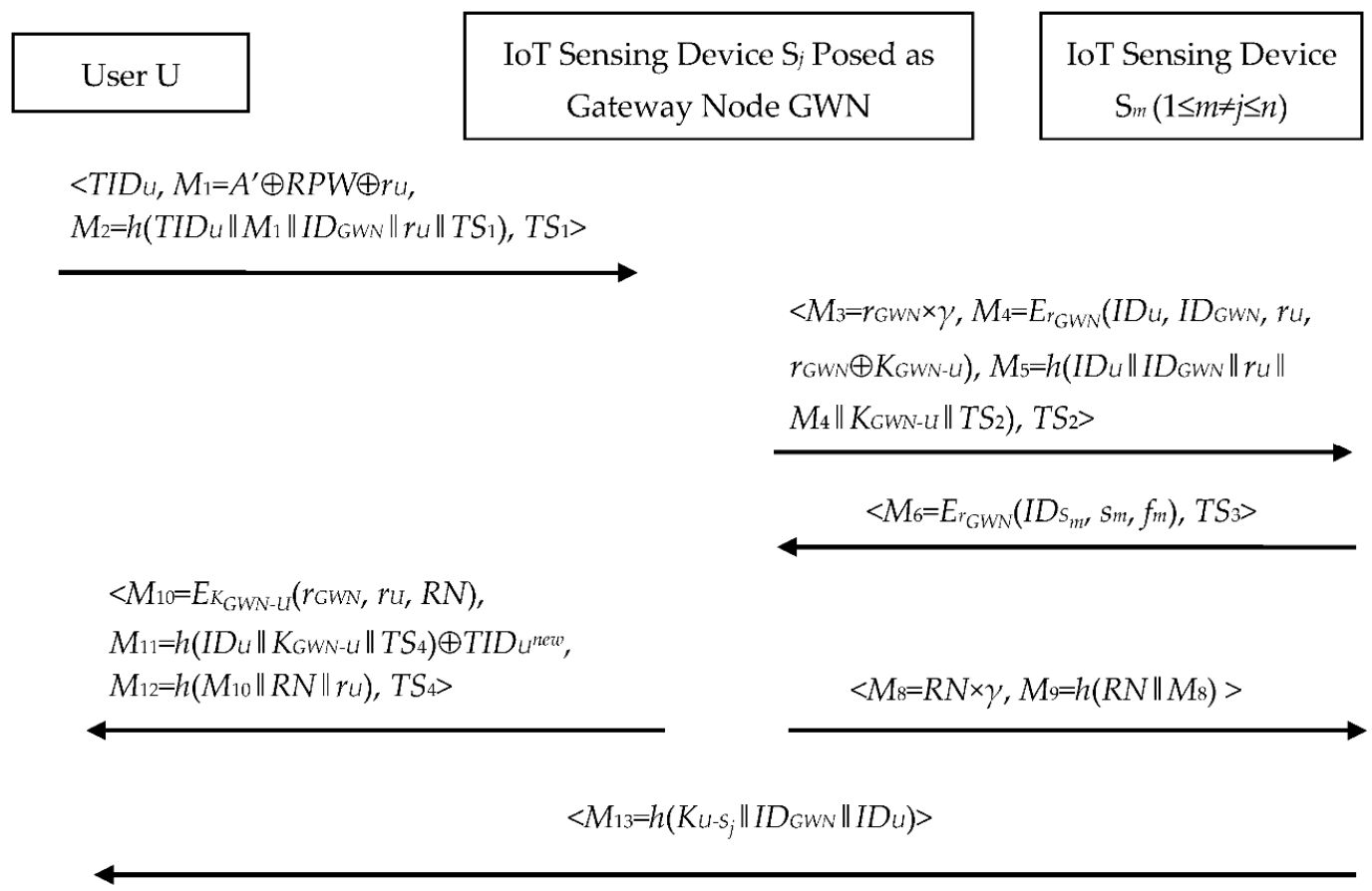

Figure 6. IoT sensing device impersonation attack on the GWN.

\subsection{Impersonation of Other IoT Sensing Devices}

If $S_{j}$ wants to impersonate any other IoT sensing device $S_{m}(1 \leq m \neq j \leq n)$, $S_{j}$ first eavesdrops on $S_{m}$ 's message $<M_{6}, T S_{3}>$ during Step 2 of the authenticated key agreement phase and computes $\left(I D_{S m}, s_{m}, f_{m}\right)=D_{r G W N}\left(M_{6}\right)$.

As shown in Figure $7, S_{j}$ can impersonate $S_{m}$ using $I D_{S m}, s_{m}, f_{m}$, and $k_{j}$ in a new authentication session. In Step 2 of the authenticated key agreement phase, $S_{j}$ recovers $r_{G W N}$ by computing $M_{3} \bmod k_{j}$. Then, $S_{j}$ uses $I D_{S m}, s_{m}, f_{m}$ to fabricate $S_{m}$ 's $M_{6}$. In Step 4 of the authenticated key agreement phase, $S_{j}$ calculates $M_{7}$ by computing $M_{8} \bmod$ 
$k_{j}$. At the end of the new authentication session, $\mathrm{U}$ believes that $\mathrm{S}_{j}$ is $\mathrm{S}_{m}$ and shares $K_{U-S j}=h\left(I D_{U}\left\|I D_{G W N}\right\| r_{G W N}\left\|r_{U}\right\| M_{7} \| K_{G W N-U}\right)$ with $S_{j}$.

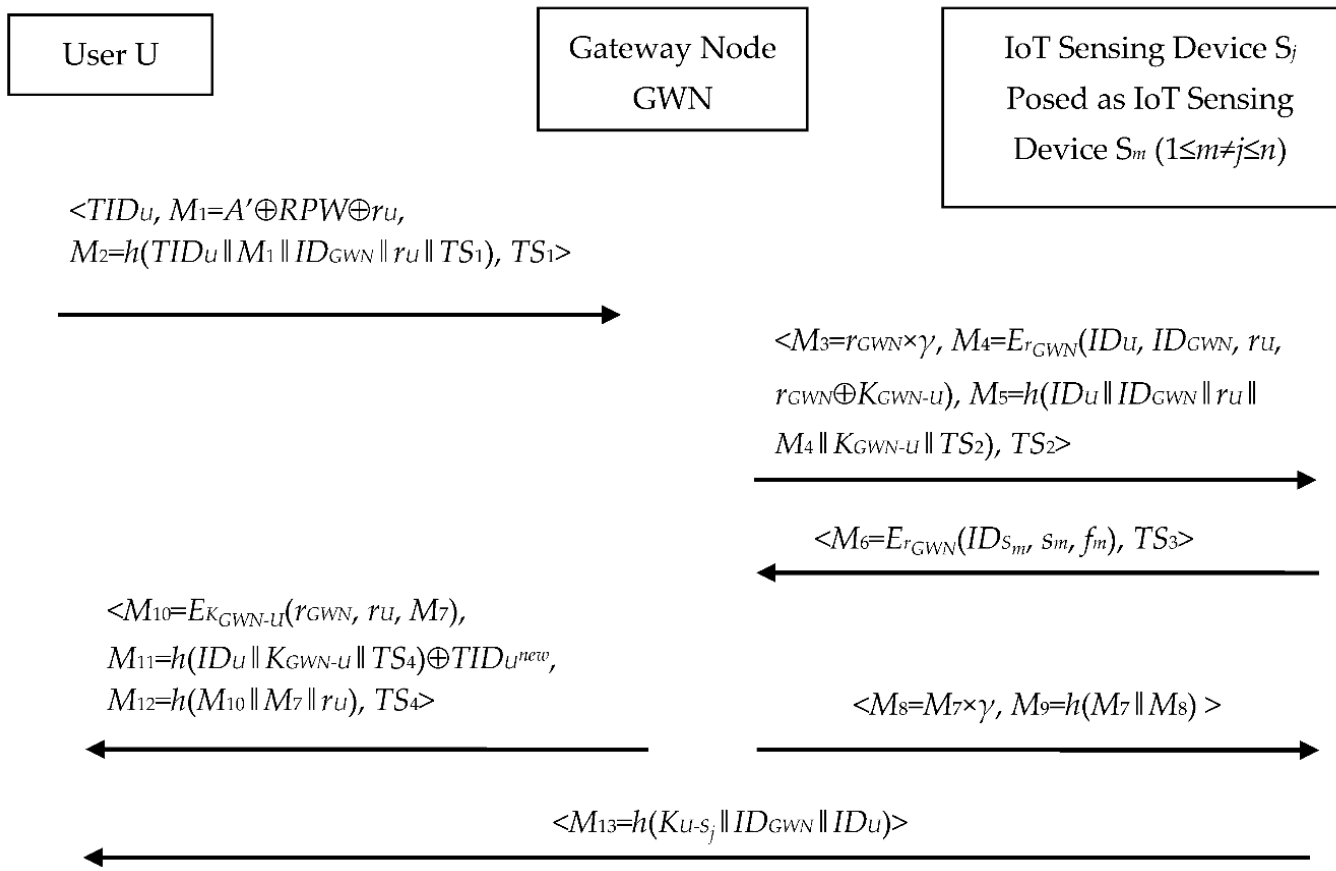

Figure 7. IoT sensing device impersonation attack on another IoT sensing device.

\subsection{Further Disscussion}

A legal but corrupt $S_{j}$ can derive U's $T I D_{U}, I D_{U}$, and $K_{G W N-U} ; G W N^{\prime} s I D_{G W N}$ and $\gamma$; and another IoT sensing device $S_{m}$ 's $I D_{S m}, s_{m}$, and $f_{m}$ from the public messages of the authentication session. Hence, $S_{j}$ successfully impersonates $U, G W N$, and $S_{m}$ by exploiting those secret parameters. To defeat the proposed attacks, Vinoth et al.'s scheme should avoid disclosing the secret parameters of other entities to $S_{j}$.

Industrial IoT sensing devices are perhaps exposed to hostile environments. An attacker may hijack and compromise industrial IoT sensing devices by physical means or Trojan horses. Once the attackers control an industrial IoT sensing device, they can subvert the industrial IoT sensing system just like the malicious user described in Section 3.4.

\section{Replay Attack}

As shown in Figure 2, we found that $S_{j}{ }^{\prime} \mathrm{s} T S_{3}$ in the message $<M_{6}, T S_{3}>$ was not protected by any cryptographic mechanism. Based on this observation of Vinoth et al.'s scheme, an outside attacker can eavesdrop on a valid message $\left\langle M_{6}, T S_{3}>\right.$ in a normal run of the authenticated key agreement phase. Then, the attacker reuses $M_{6}$ and attaches the current timestamp $T S_{3}{ }^{*}$ to impersonate $S_{j}$. Figure 8 describes this replay attack on Vinoth et al.'s scheme. After the replay attack, GWN believes that the attacker is $S_{j}$, although the attacker does not know any secret of $S_{j}$. Meanwhile, $U$ does not authenticate the attacker as $S_{j}$. Note that GWN actually finishes its session in Step 3 of the authenticated key agreement phase and updates U's temporary identity. As a result, GWN updates the old $T I D_{U}$ to a new $T I D_{U}{ }^{\text {new }}$, but $U$ still keeps the old $T I D_{U}$. This means that $U$ cannot log into the deployed network anymore, because during Step 1 of the authenticated key agreement phase, GWN fails to retrieve $I D_{U}$ and $K_{G W N-U}$ according to U's old $T I D_{U}$. For the industrial IoT sensing system, the legal user faces denial of service once the attacker implements the replay attack. 


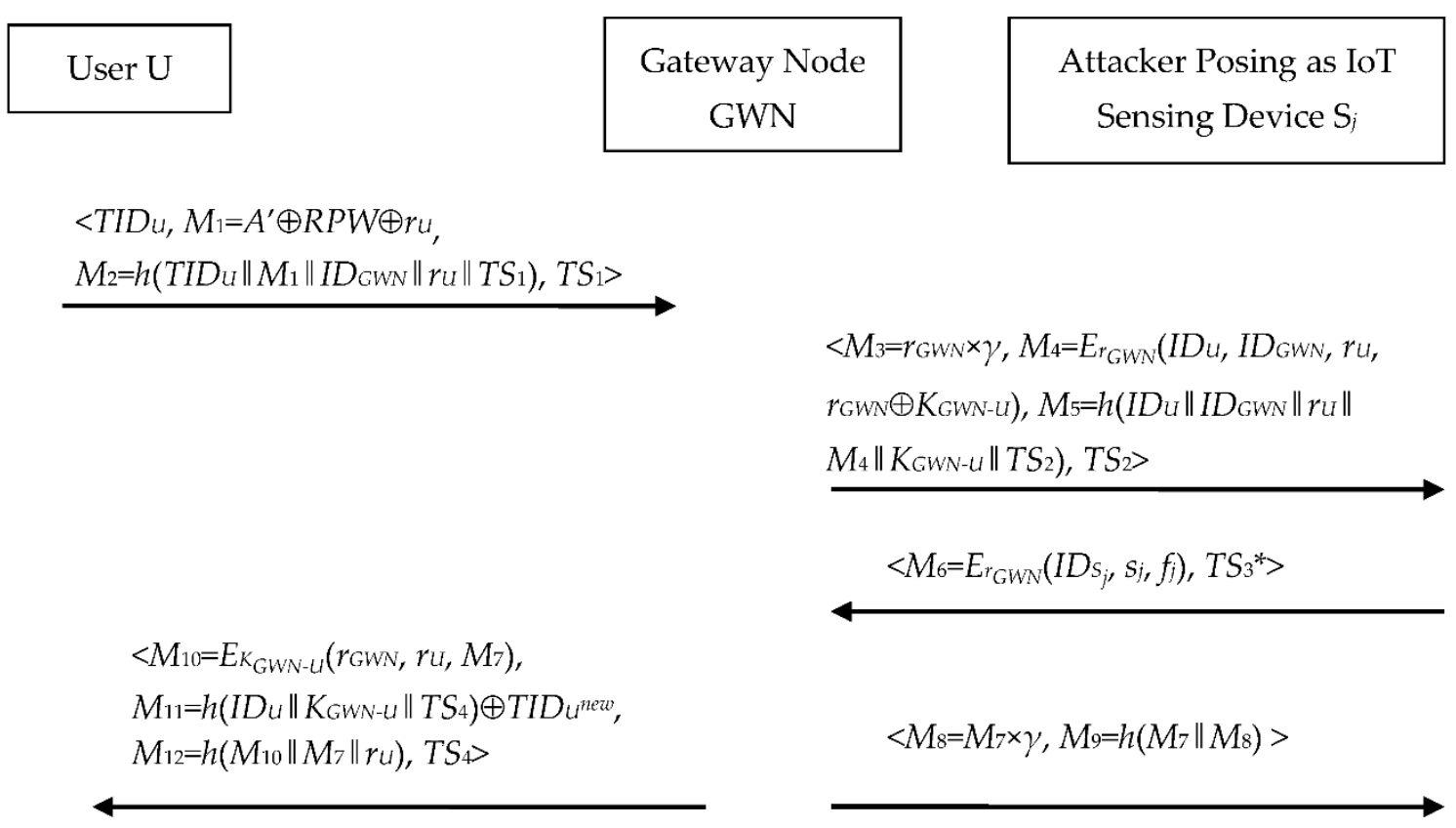

Figure 8. Replay attack.

To fix this vulnerability, we suggest that $T S_{3}$ should be protected by the cryptographic mechanism. For example, $S_{j}$ could compute $M_{6}=E_{r G W N}\left(I D_{S_{j}}, s_{j}, f_{j}, T S_{3}\right)$ instead of $M_{6}=E_{r G W N}\left(I D_{S_{j}}, s_{j}, f_{j}\right)$ in Step 2 of the authenticated key agreement phase.

\section{Desynchronization Attack}

In Vinoth et al.'s scheme, $U$ and GWN keep the same $T I D_{U}$ to authenticate each other. Hence, as shown in Figure 9, an outside attacker can block the message $<M_{10}, M_{11}, M_{12}$, $T S_{4}>$ in Step 3 of the authenticated key agreement phase and instead send the message $<M_{10}, R N, M_{12}, T S_{4}{ }^{*}>$ to U. Here, $T S_{4}{ }^{*}$ is the attacker's current timestamp. During Step 5 of the authenticated key agreement phase, $\mathrm{U}$ confirms the freshness of $T S_{4}{ }^{*}$ in the fabricated message $\left\langle M_{10}, R N, M_{12}, T S_{4}^{*}\right\rangle$, decrypts $r_{U}$ from $M_{10}$, and successfully verifies $r_{U}$ and $M_{12}$. $\mathrm{U}$ also computes $K_{U-S j}=h\left(I D_{U}\left\|I D_{G W N}\right\| r_{G W N}\left\|r_{U}\right\| M_{7} \| K_{G W N-U}\right)$ and verifies $M_{13}$. Then, $\mathrm{U}$ further updates $T I D_{U}$ to $T I D_{U}{ }^{\text {new }}=h\left(I D_{U}\left\|K_{G W N-U}\right\| T S_{4}^{*}\right) \oplus R N$. The $T I D_{U}{ }^{\text {new }}$ computed by $\mathrm{U}$ is not equal to the $T I D_{U}{ }^{\text {new }}$ generated by GWN during Step 3 of the authenticated key agreement phase. This causes the failure to authenticate in the subsequent runs of Vinoth et al.'s scheme, though $\mathrm{U}, \mathrm{GWN}$, and $\mathrm{S}_{j}$ are all legal and honest.

The attacker randomly changes $M_{11}$ because Vinoth et al.'s scheme does not check the authenticity of $M_{11}$. To overcome the desynchronization attack, our suggestion is to apply the message authentication code algorithm for $M_{11}$. Where industrial IoT sensing applications are concerned, this desynchronization attack has the same negative impact as the replay attack discussed in Section 5. 


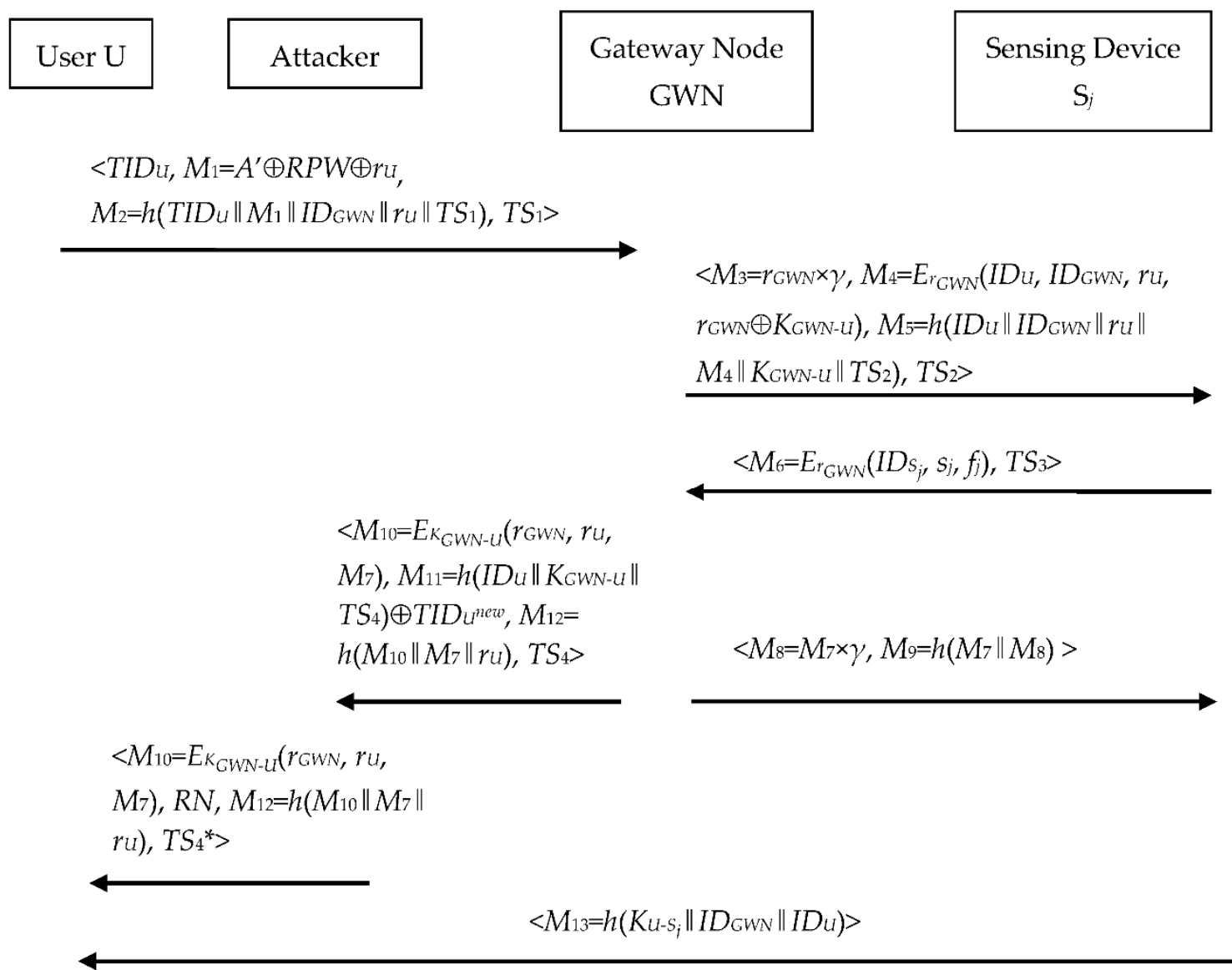

Figure 9. Desynchronization attack.

\section{Weakness of User Privacy}

In the authenticated key agreement scheme, user privacy guarantees that the attacker cannot derive the user's identity from the transmitted messages of the authentication sessions. This is called user anonymity. Moreover, the attacker also fails to link two different authentication sessions to the same user. This is called untraceability. User privacy is a concern in industrial IoT sensing applications, as users' private data can be leaked and misused if a factory deployed with IoT sensing devices is subjected to cyberattacks. For example, users' presence or absence at the industrial control room can be revealed simply by observing authentication sessions.

Vinoth et al. claimed that their scheme supported both user anonymity and untraceability because it employed the temporal $T I D_{U}$ to hide U's long-term $I D_{U}$. Furthermore, the symmetric encryption algorithm and cryptographic hash function were utilized to protect U's $I D_{U}$. In Section 3.2, we show that $\mathrm{U}_{a}$ can attain any other target user U's $I D_{U}$, $K_{G W N-U}$, and $T I D_{U}{ }^{\text {new }}$. Hence, when $\mathrm{U}_{a}$ finds $T I D_{U}{ }^{\text {new }}$ in the message $<T I D_{U}, M_{1}, M_{2}$, $T S_{1}>$ during Step 2 of the login phase, $\mathrm{U}_{a}$ knows that $\mathrm{U}$ is running a session. Then, $\mathrm{U}_{a}$ can eavesdrop on the message $<M_{10}, M_{11}, M_{12}, T S_{4}>$ in Step 3 of the authenticated key agreement phase and synchronously update U's new temporal $T I D_{U}{ }^{\text {new }}$ by computing $h\left(I D_{U}\left\|K_{G W N-U}\right\| T S_{4}\right) \oplus M_{11}$. As a result, $\mathrm{U}_{a}$ can track any $\mathrm{U}$ all the time. $\mathrm{S}_{j}$ also can track any target user U. $S_{j}$ first derives U's $T I D_{U}, I D_{U}$, and $K_{G W N-U}$ as discussed in Section 4.1. Then, $S_{j}$ uses $T I D_{U}$ to identify $U$, eavesdrops on the message $<M_{10}, M_{11}, M_{12}, T S_{4}>$ during Step 3 of the authenticated key agreement phase and updates $T I D_{U}$ just like U. In conclusion, Vinoth et al.'s scheme fails to provide the user privacy protection.

Vinoth et al.'s scheme suffers from weak user privacy because it is vulnerable to user and IoT sensing device impersonation attacks. Vinoth et al.'s scheme could pro- 
vide better user privacy if both user and IoT sensing device impersonation attacks are repaired correctly.

\section{Conclusions and Future Work}

In Vinoth et al.'s scheme, the user and multiple IoT sensing devices negotiate a secret session key via a group key, i.e., $r_{G W N}$. This novel design improves the efficiency of Vinoth et al.'s scheme. It is a desirable feature of the IoT sensing applications. Hence, we study Vinoth et al.'s scheme in aspects of security and privacy. Although Vinoth et al.'s scheme proved secure under the Canetti-Krawczyk threat model [40], we still revealed several serious security and privacy vulnerabilities in the scheme. In addition, Vinoth et al.'s scheme employs random numbers such as $r_{U}$ and $r_{G W N}$ and timestamps such as $T S_{1}, T S_{2}$, $T S_{3}$, and $T S_{4}$ at the same time. It is widely known that random numbers and timestamps are both used to defeat reply attacks and ensure the freshness of the message. From the perspective of applications, the use of both random numbers and timestamps increases the complexity of the authentication system and brings greater security risk. Therefore, it would be best to adopt only one of them in an authenticated key agreement scheme.

It is still a challenge to design a robust and efficient authenticated key agreement scheme for IoT sensing applications. One avenue for future work is to formulate a communication model appropriate for defining authentication and key agreement goals and present the definitions of security and privacy under the communication model. The results of our analysis of Vinoth et al.'s scheme can provide a reference for these definitions. Another avenue for future work is to develop an authenticated key agreement scheme that not only satisfies our formal definitions but also achieves high efficiency. In [41], Bellare and Rogaway proposed a security definition, a protocol, and a proof for secure session key distribution with the trust three-party case. One feasible idea is to extend Bellare and Rogaway's definition and protocol for IoT sensing models. We expect that this will require a great deal of research work to accomplish.

Funding: The work of Da-Zhi Sun was supported in part by the National Natural Science Foundation of China under Grant No. 61872264. The APC was funded by the National Natural Science Foundation of China under Grant No. 61872264.

Data Availability Statement: No new data were created or analyzed in this study. Data sharing is not applicable to this article.

Acknowledgments: The author would like to thank the editor and the reviewers for their valuable suggestions and comments.

Conflicts of Interest: The author declares no conflict of interest. The funders had no role in the design of the study; in the collection, analyses, or interpretation of data; in the writing of the manuscript; or in the decision to publish the results.

\section{References}

1. Hirman, M.; Benesova, A.; Sima, K.; Steiner, F.; Tupa, J. Design, fabrication and risk assessment of IoT unit for products manufactured in industry 4.0 factory. Procedia Manuf. 2020, 51, 1178-1183. [CrossRef]

2. Macioszek, E.; Kurek, A. Extracting road traffic volume in the city before and during COVID-19 through video remote sensing. Remote Sens. 2021, 13, 2329. [CrossRef]

3. Hassija, V.; Chamola, V.; Saxena, V.; Jain, D.; Goyal, P.; Sikdar, B. A survey on IoT security: Application areas, security threats, and solution architectures. IEEE Access 2019, 7, 82721-82743. [CrossRef]

4. Vinoth, R.; Deborah, L.J.; Vijayakumar, P.; Kumar, N. Secure multifactor authenticated key agreement scheme for industrial IoT. IEEE Internet Things J. 2021, 8, 288-296. [CrossRef]

5. Kumari, S.; Khan, M.K.; Atiquzzaman, M. User authentication schemes for wireless sensor networks: A review. Ad Hoc Netw. 2015, 27, 159-194. [CrossRef]

6. Singh, D.; Kumar, B.; Singh, S.; Chand, S. Evaluating authentication schemes for real-time data in wireless sensor network. Wirel. Pers. Commun. 2020, 114, 629-655. [CrossRef]

7. Sun, D.Z.; Li, J.X.; Feng, Z.Y.; Cao, Z.F.; Xu, G.Q. On the security and improvement of a two-factor user authentication scheme in wireless sensor networks. Pers. Ubiquitous Comput. 2013, 17, 895-905. [CrossRef] 
8. Wang, D.; Wang, P. Understanding security failures of two-factor authentication schemes for real-time applications in hierarchical wireless sensor networks. Ad Hoc Netw. 2014, 20, 1-15. [CrossRef]

9. Jiang, Q.; Ma, J.; Lu, X.; Tian, Y.L. An efficient two-factor user authentication scheme with unlinkability for wireless sensor networks. Peer-Peer Netw. Appl. 2015, 8, 1070-1081. [CrossRef]

10. Wei, F.; Zhang, R.; Shen, J. A Provably Secure Two-Factor Authenticated Key Exchange Protocol for Wireless Sensor Networks Based on Authenticated Encryption. In Lecture Notes on Data Engineering and Communications Technologies, Proceedings of the 11th International Conference on Advances on Broad-Band Wireless Computing, Communication and Applications (BWCCA 2016), Asan, Korea, 5-7 November 2016; Barolli, L., Xhafa, F., Yim, K., Eds.; Springer: Cham, Switzerland, 2017; Volume 2, pp. 849-855.

11. $\mathrm{Wu}, \mathrm{F}$; $\mathrm{Xu}, \mathrm{L} . \mathrm{L} . ; \mathrm{Kumari}, \mathrm{S} . ; \mathrm{Li}, \mathrm{X}$. A new and secure authentication scheme for wireless sensor networks with formal proof. Peer-Peer Netw. Appl. 2017, 10, 16-30. [CrossRef]

12. Wu, F.; Li, X.; Sangaiah, A.K.; Xu, L.L.; Kumari, S.; Wu, L.X.; Shen, J. A lightweight and robust two-factor authentication scheme for personalized healthcare systems using wireless medical sensor networks. Future Gener. Comput. Syst. 2018, 82, 727-737. [CrossRef]

13. Chandrakar, P. A secure remote user authentication protocol for healthcare monitoring using wireless medical sensor networks. Int. J. Ambient Comput. Intell. 2019, 10, 6. [CrossRef]

14. Kaur, D.; Kumar, D. Cryptanalysis and improvement of a two-factor user authentication scheme for smart home. J. Inf. Secur. Appl. 2021, 58, 102787.

15. Qi, M.P.; Chen, J.H. Secure authenticated key exchange for WSNs in IoT applications. J. Supercomput. 2021. [CrossRef]

16. Das, A.K. An efficient and novel three-factor user authentication scheme for large-scale heterogeneous wireless sensor networks. Int. J. Commun. Netw. Distrib. Syst. 2015, 15, 22-60. [CrossRef]

17. Das, A.K. A secure and robust temporal credential-based three-factor user authentication scheme for wireless sensor networks. Peer-Peer Netw. Appl. 2016, 9, 223-244. [CrossRef]

18. Wang, C.Y.; Xu, G.A.; Sun, J. An enhanced three-factor user authentication scheme using elliptic curve cryptosystem for wireless sensor networks. Sensors 2017, 17, 2946. [CrossRef] [PubMed]

19. Wu, F.; Xu, L.L.; Kumari, S.; Li, X. An improved and provably secure three-factor user authentication scheme for wireless sensor networks. Peer-Peer Netw. Appl. 2018, 11,1-20. [CrossRef]

20. Shin, S.; Kwon, T. A lightweight three-factor authentication and key agreement scheme in wireless sensor networks for smart homes. Sensors 2019, 19, 2012. [CrossRef]

21. Luo, H.G.; Wen, G.J.; Su, J. Lightweight three factor scheme for real-time data access in wireless sensor networks. Wirel. Netw. 2020, 26, 955-970. [CrossRef]

22. Jabbari, A.; Mohasef, J.B. Improvement of a user authentication scheme for wireless sensor networks based on internet of things security. Wirel. Pers. Commun. 2021, 116, 2565-2591. [CrossRef]

23. Jiang, Q.; Kumar, N.; Ma, J.F.; Shen, J.; He, D.B.; Chilamkurti, N. A privacy-aware two-factor authentication protocol based on elliptic curve cryptography for wireless sensor networks. Int. J. Netw. Manag. 2017, 27, e1937. [CrossRef]

24. Adavoudi-Jolfaei, A.; Ashouri-Talouki, M.; Aghili, S.F. Lightweight and anonymous three-factor authentication and access control scheme for real-time applications in wireless sensor networks. Peer-Peer Netw. Appl. 2019, 12, 43-59. [CrossRef]

25. Lu, Y.R.; Xu, G.Q.; Li, L.X.; Yang, Y.X. Anonymous three-factor authenticated key agreement for wireless sensor networks. Wirel. Netw. 2019, 25, 1461-1475. [CrossRef]

26. Sadri, M.J.; Asaar, M.R. A lightweight anonymous two-factor authentication protocol for wireless sensor networks in internet of vehicles. Int. J. Commun. Syst. 2020, 33, e4511. [CrossRef]

27. Far, H.A.N.; Bayat, M.; Das, A.K.; Fotouhi, M.; Pournaghi, S.M.; Doostari, M.A. LAPTAS: Lightweight anonymous privacypreserving three-factor authentication scheme for WSN-based IIoT. Wirel. Netw. 2021, 27, 1389-1412.

28. Das, A.K.; Sutrala, A.K.; Kumari, S.; Odelu, V.; Wazid, M.; Li, X. An efficient multi-gateway-based three-factor user authentication and key agreement scheme in hierarchical wireless sensor networks. Secur. Commun. Netw. 2016, 9, 2070-2092. [CrossRef]

29. Amin, R.; Biswas, G.P. A secure light weight scheme for user authentication and key agreement in multi-gateway based wireless sensor networks. Ad Hoc Netw. 2016, 36, 58-80. [CrossRef]

30. Wu, F.; Xu, L.L.; Kumari, S.; Li, X.; Shen, J.; Choo, K.K.R.; Wazid, M.; Das, A.K. An efficient authentication and key agreement scheme for multi-gateway wireless sensor networks in IoT deployment. J. Netw. Comput. Appl. 2017, 89, 72-85. [CrossRef]

31. Sutrala, A.K.; Das, A.K.; Reddy, A.G.; Vasilakos, A.V.; Rodrigues, J.J.P.C. On the design of secure user authenticated key management scheme for multigateway-based wireless sensor networks using ECC. Int. J. Commun. Syst. 2018, 31, e3514. [CrossRef]

32. Guo, H.; Gao, Y.; Xu, T.G.; Zhang, X.Y.; Ye, J.F. A secure and efficient three-factor multi-gateway authentication protocol for wireless sensor networks. Ad Hoc Netw. 2019, 95, 101965. [CrossRef]

33. Lee, J.; Yu, S.; Park, K.; Park, Y.; Park, Y. Secure three-factor authentication protocol for multi-gateway IoT environments. Sensors 2019, 19, 2358. [CrossRef] [PubMed]

34. Xu, L.L.; Wu, F. A lightweight authentication scheme for multi-gateway wireless sensor networks under IoT conception. Arab. J. Sci. Eng. 2019, 44, 3977-3993. [CrossRef]

35. Wang, D.; Hong, S.H.; Wang, Q.X. Revisiting a multifactor authentication scheme in industrial IoT. Secur. Commun. Netw. 2021, 2021, 9995832. [CrossRef] 
36. Vinoth, R.; Deborah, L.J. An efficient key agreement and authentication protocol for secure communication in industrial IoT applications. J. Ambient Intell. Humaniz. Comput. 2021. [CrossRef]

37. Gupta, M.; Chaudhari, N.S. Anonymous two factor authentication protocol for roaming service in global mobility network with security beyond traditional limit. Ad Hoc Netw. 2019, 84, 56-67. [CrossRef]

38. Wang, F.F.; Xu, G.A.; Gu, L.Z. A secure and efficient ECC based anonymous authentication protocol. Secur. Commun. Netw. 2019, 2019, 4656281. [CrossRef]

39. Jiang, Q.; Zhang, N.; Ni, J.B.; Ma, J.F.; Ma, X.D.; Choo, K.K.R. Unified biometric privacy preserving three-factor authentication and key agreement for cloud-assisted autonomous vehicles. IEEE Trans. Veh. Technol. 2020, 69, 9390-9401. [CrossRef]

40. Canetti, R.; Krawczyk, H. Analysis of Key-Exchange Protocols and Their Use for Building Secure Channels. In Lecture Notes in Computer Science, Proceedings of the International Conference on the Theory and Applications of Cryptographic Techniques (EUROCRYPT 2001), Innsbruck, Austria, 6-10 May 2001; Pfitzmann, B., Ed.; Springer: Berlin/Heidelberg, Germany, 2001; Volume 2045, pp. 453-474.

41. Bellare, M.; Rogaway, P. Provably Secure Session Key Distribution-The Three Party Case. In Proceedings of the 27th ACM Symposium on the Theory of Computing (STOC'95), Las Vegas, NV, USA, 29 May-1 June 1995; ACM: New York, NY, USA, 1995; pp. 57-66. 